\title{
MicroRNA-4458 suppresses migration and epithelial-mesenchymal transition via targeting HMGAI in non-small-cell lung cancer cells
}

This article was published in the following Dove Medical Press journal: Cancer Management and Research

Yu Ma

Xuena Li

Song Chen

Bulin Du

Yaming $\mathrm{Li}$

Department of Nuclear Medicine, The First Hospital of China Medical University, Shenyang II000I, Liaoning Province, China
Correspondence: Yaming Li Department of Nuclear Medicine, The First Hospital of China Medical University, No. I 55 North Nanjing Street, Heping District, Shenyang I I000I, Liaoning Province, China

Tel +86248328 2412

Emailymli200।@I63.com
Purpose: Increasing studies have shown that microRNA-4458 (miR-4458) is associated with human cancer progression. However, the molecular mechanism of miR-4458 in non-small-cell lung cancer (NSCLC) remains largely unknown. This study aims to reveal the biological function of miR-4458 in NSCLC.

Materials and methods: The expression of miR-4458 in NSCLC cells was evaluated by qRT-PCR. Cell proliferation and migration assay were carried out in vitro after transfection. A luciferase reporter and Western blot assay were performed to identify the functional target of miR-4458.

Results: The study indicated that miR-4458 was markedly downregulated in NSCLC cells Overexpression of miR-4458 strongly reduced the proliferation and migration in NSCLC cell lines. In addition, miR-4458 inhibited the progression of migration and epithelial-mesenchymal transition (EMT) through the PI3K/AKT pathway. Luciferase report assay demonstrated that HMGA1 was a target gene for miR-4458.

Conclusion: The results indicate that miR-4458 participated in the process of migration and EMT via directly targeting HMGA1 and miR-4458 might be a potential novel therapeutic target in NSCLC.

Keywords: HMGA1, migration, epithelial-mesenchymal transition, miR-4458, NSCLC

\section{The plain language summary}

This study aims to reveal the biological mechanism of microRNA-4458 (miR-4458) in nonsmall-cell lung cancer (NSCLC). miR-4458 was markedly downregulated in NSCLC cells by qRT-PCR. Overexpression of miR-4458 strongly reduced the proliferation and migration in NSCLC cell lines. In addition, miR-4458 inhibited the progression of migration and epithelial-mesenchymal transition (EMT) through the PI3K/AKT pathway. Luciferase report assay demonstrated that HMGA1 was a target gene for miR-4458. The results indicate that miR-4458 participated in the process of migration and EMT via directly targeting HMGA1.

\section{Introduction}

Lung cancer is one of the leading causes of cancer-related deaths worldwide. ${ }^{1}$ Nonsmall-cell lung cancer (NSCLC) accounts for $\sim 80 \%$ of lung cancer. ${ }^{2}$ Although considerable advancements have been made in diagnosis and targeted therapy for NSCLC, the prognosis is still poor. ${ }^{3,4}$ Therefore, it is crucial to have a better understanding of the exact mechanism for the development and progression of NSCLC, which could provide more individualized and effective therapeutic strategies for NSCLC patients. 
miRNAs are a class of small (22-24 nucleotides) ncRNAs that play a pivotal role in the diagnosis and prognosis of malignant neoplasm..$^{5-7}$ Previous studies have already found that miRNAs could inhibit the transcription of target mRNAs via binding to complementary 3 '-UTR. Emerging evidence indicates that microRNA-4458 (miR-4458) plays an important role in different cell processes, including proliferation, cell cycle, and glycolysis in hepatocellular

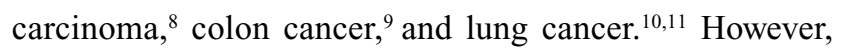
the molecular mechanism of miR-4458 in NSCLC has not been fully understood. Therefore, the understanding of the biological activities utilized by miR-4458 in NSCLC is urgently required.

The HMGA1 serves as a regulator of the chromatin structure via direct binding to A/T-rich DNA sequences. ${ }^{12}$ Studies find that HMGA1 plays a carcinogenic role in various cancer types, such as thyroid cancer, ${ }^{13}$ breast cancer, ${ }^{14}$ and lung cancer. ${ }^{15}$ Accumulating evidence shows that HMGA1 is associated with biological processes of cell proliferation, cell cycle, and metastasis. ${ }^{16,17}$ Moreover, overexpression of HMGA1 leads to the promotion of epithelial-mesenchymal transition (EMT) in basal-like breast cancer. ${ }^{18}$ In addition, HMGA1 could be regulated by miRNAs, such as miR-26a ${ }^{19}$ and miR- $625 .{ }^{20}$ However, its role and the molecular mechanism in NSCLC still remain obscure.

In the present study, we demonstrated that miR-4458 inhibited proliferation and migration in NSCLC cells. It was shown that miR-4458 suppressed the progression of migration and EMT through the PI3K/AKT pathway. Furthermore, we explored and validated HMGA1 as a direct target of miR-4458. Thus, our results suggest that miR-4458 might be a potential therapeutic target in NSCLC.

\section{Materials and methods}

\section{Cell culture and transfection}

A549, H1299, HCC827, PC9, HBE, 293 T cell lines were purchased from American Type Culture Collection (Manassas, VA, USA). All cells were cultured at $37^{\circ} \mathrm{C}$ in an incubator with $5 \% \mathrm{CO}_{2}$. miR-4458 mimics (mimics), negative control (NC), miR-4458 inhibitor (inhibitor), and inhibitor negative control (inhibitor NC) were used (RiboBio, Guangzhou, China) for the overexpression and knockdown of miR4458. The si-HMGA1 was conducted for the knockdown of HMGA1 and si-NC was used as the control. Transfection of cells was performed using riboFECT ${ }^{\mathrm{TM}} \mathrm{CP}$ Transfection Agent (RiboBio, Guangzhou, China) with a $100 \mathrm{nM}$ concentration following the manufacturer's protocol.

\section{qRT-PCR analysis}

The total RNA was extracted from cultured cells using TRIzol (Thermo Fisher Scientific, Waltham, MA, USA). The expression level of miR-4458 was assessed by Hairpin-it ${ }^{\mathrm{TM}}$ microRNA RT-PCR Quantitation Kit (Genepharma, Shanghai, China) while the mRNA expression was measured by a SYBR Premix Ex Taq II kit (TaKaRa, Dalian, China). $\beta$-actin and U6 were used as an internal control. miR-4458 and mRNA expression was evaluated using Light Cycler 480 System II (Roche).

\section{EdU assay}

After 48 hours of transfection, 5-ethynyl-2'-deoxyuridine (Edu) was added into A549 and H1299 cells with 2-hour incubation at $37^{\circ} \mathrm{C}$. Then, the cell proliferation assay was performed by Cell-Light EdU Apollo ${ }^{\circledR} 57$ In Vitro Imaging Kit (RiboBio, Guangzhou, China) according to the manufacturer's protocol.

\section{Cell counting kit-8 (CCK-8) assay}

Cells were seeded into 96 -well plates $\left(2 \times 10^{3}\right.$ cells per well) after 24 hours of transfection. Cell proliferation assay was assessed by CCK-8 assay (Dojindo, Kumamoto, Japan) using a microplate reader (Thermo Fisher Scientific) at 450 $\mathrm{nm}$ wavelength. The assay was repeated six times at each time point.

\section{Colony formation assay}

For colony formation assay, A549 and H1299 cells transfected with miR-4458 mimics and inhibitors were seeded into 6-well plates with a density of 200 cells per well. After culturing in the cell incubator $\left(37^{\circ} \mathrm{C}, 5 \% \mathrm{CO}_{2}\right)$ for 14 days, the cells were fixed in $70 \%$ ethanol and stained by crystal violet.

\section{Cell migration assay}

In order to detect the migratory capability, $2 \times 10^{5}$ cells were seeded into a transwell apparatus (Corning, NY, USA) with the serum-free medium. Relatively, medium containing $10 \%$ serum functioned as a chemoattractant in the lower chamber. After incubation for 12 hours, the stained cells were photographed and counted by a microscope.

\section{Wound healing assay}

A549 and H1299 cells transfected with miR-4458 mimics, inhibitors, and si-HMGA1 were seeded into six-well plates to perform wound healing assay. When the fusion of cells reached above $90 \%$, the scratch was marked by using a standard $10 \mu \mathrm{L}$ pipette tip at the middle of the well. Cells 
were further cultured with serum-free medium for 24 hours. The inverted microscope was used to observe the healing of the wound.

\section{Western blotting analysis}

Cells were harvested and lysed by RIPA buffer (Beyotime, Shanghai, China) after 48 hours of transfection. After the separation of protein using $10 \%$ SDS-PAGE and the transfer using polyvinylidene fluoride membrane, the antibodies were incubated at $4^{\circ} \mathrm{C}$ overnight after blocking for 2 hours with 5\% skim milk. The antibodies against HMGA1, E-cadherin, $\mathrm{N}$-cadherin, Snail, AKT, and p-AKT were obtained from Abcam (Cambridge, UK) while $\beta$-actin antibody served as a loading control. An ECL Advanced Western Blot Detection Kit (Thermo Fisher Scientific) was used to visualize the specific protein bands. The Image Lab software was used for analyzing the results from Western blot. The experiments were repeated three times.

\section{Bioinformatics analysis}

Publicly available databases were used to predict potential targets of miR-4458, including TargetScan (www.targetscan. org/), TarBase (http://diana.imis.athena-innovation.gr/ DianaTDian/index.php? $\mathrm{r}=$ tarbase/index), and miRwalk (http://mirwalk.umm.uni-heidelberg.de/). We explored common target genes from three databases and then carried out the analysis of Gene Ontology (GO) and pathway enrichment using Funrich software. In order to explore more possible targets, the expression profile data of lung adenocarcinoma (LUAD) from the The Cancer Genome Atlas (TCGA) database including 73 cancer samples and 59 normal samples were analyzed. GSE32863 and GSE10072 datasets were explored to define the expression of HMGA1 between LUAD and normal group by Oncomine database (www. oncomine.org/resource/login.html). For the understanding of prognostic information, the free available Kaplan-Meier plotter database (www.kmplot.com) was used to analyze the overall survival (OS) of HMGA1. Cancer Cell Line Encyclopedia (CCLE) database (https://portals.broadinstitute. org/ccle) was used to visualize the expression of HMGA1 in lung cancer cell lines.

\section{Dual luciferase reporter assay}

First, cells $\left(1.5 \times 10^{4}\right)$ were plated on 96 -well plates. Next, cells were cotransfected with different combinations of miR-4458, miR-NC, h-HMGA1-wild type (WT), and h-HMGA1-mutant type (MUT) after 24 hours. Finally, the relative luciferase signals were assessed after 48 hours using the Dual Luciferase
Reporter Assay Kit (Promega Corporation, Fitchburg, WI, USA).

\section{Statistical analyses}

All statistical analysis was carried out using GraphPad Prism 6.0 software (La Jolla, CA, USA). Results were expressed as the mean \pm SD among triplicate samples. One-way ANOVA or two-tailed Student's $t$-test was applied to evaluate statistical differences. $P<0.05$ was considered to be statistically significant.

\section{Results}

\section{miR-4458 inhibited cell proliferation and colony formation in NSCLC cells}

qRT-PCR was employed to investigate the expression of miR-4458. As expected, miR-4458 was significantly downregulated in four NSCLC cell lines (A549, H1299, HCC827, and PC9) compared with one normal lung cell (HBE, Figure 1A). Higher expression of miR-4458 was discovered in NSCLC cells transfected with miR-4458 mimics. Meanwhile, the expression of miR-4458 was lower in NSCLC cells transfected with miR-4458 inhibitor (Figure 1B). In order to monitor whether miR-4458 could influence the proliferation in NSCLC cells, we performed Edu and CCK-8 assay. The results of Edu assay indicated that overexpression of miR4458 inhibited proliferation. In contrast, downregulation of miR-4458 enhanced cell growth of NSCLC cells (Figure 1C). Furthermore, CCK-8 assay confirmed that miR-4458 overexpression suppressed the proliferation rate, whereas miR-4458 knockdown increased growth of NSCLC cells compared with the NC group (Figure 1D). Consistent with these findings, colony formation ability was reduced with the overexpression of miR-4458 and enhanced with the knockdown of miR-4458 (Figure 1E). In conclusion, these results demonstrated that miR-4458 inhibited cell proliferation and colony formation in NSCLC cells.

\section{miR-4458 inhibited migration and the EMT process in NSCLC cells}

In order to explore the migratory capability of miR-4458 in NSCLC, we carried out transwell migration assay. The results showed that miR-4458 overexpression significantly inhibited migration in NSCLC cells. Moreover, knockdown of miR-4458 led to an increase in migration compared with the NC group (Figure 2A). Furthermore, wound healing assay confirmed that miR-4458 overexpression suppressed migration, whereas miR-4458 knockdown increased migra- 


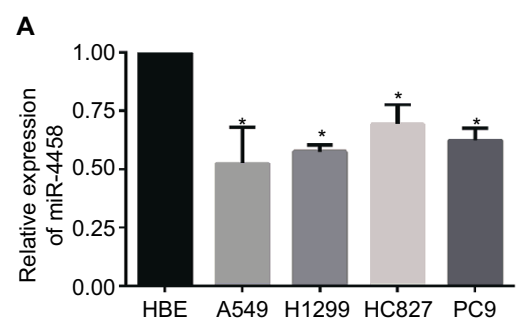

C

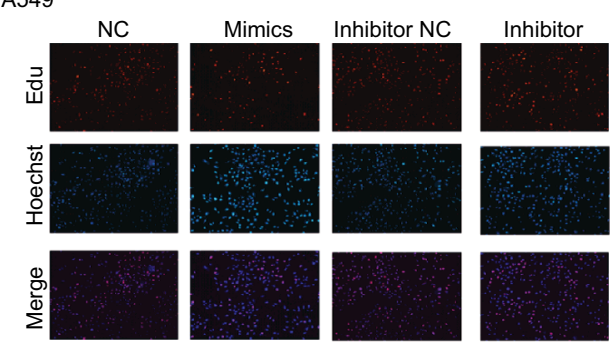

H1299
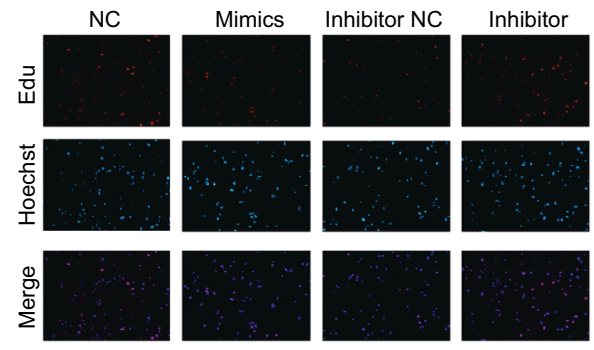

D

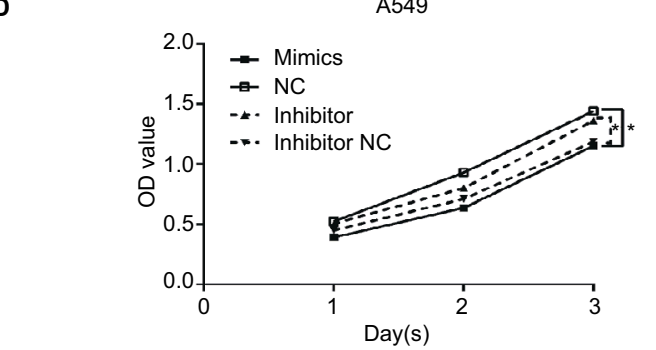

E

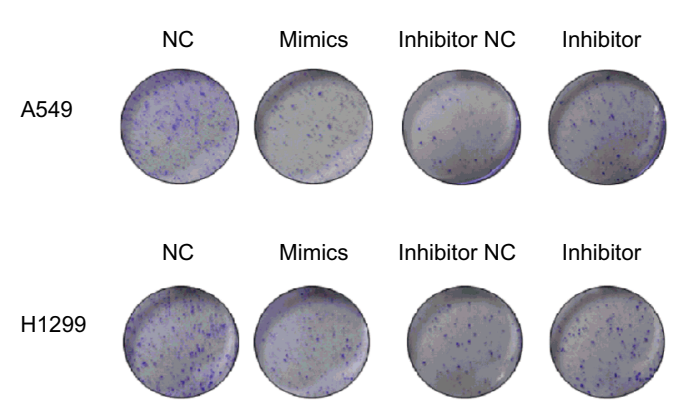

B
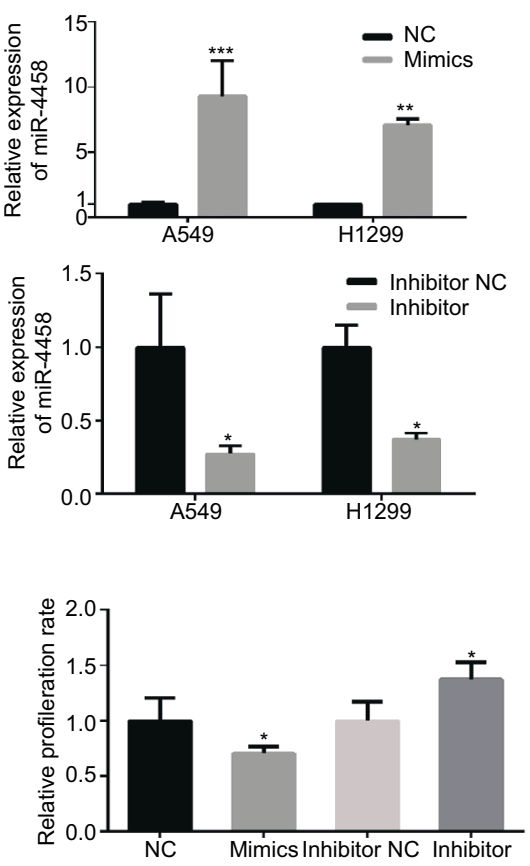
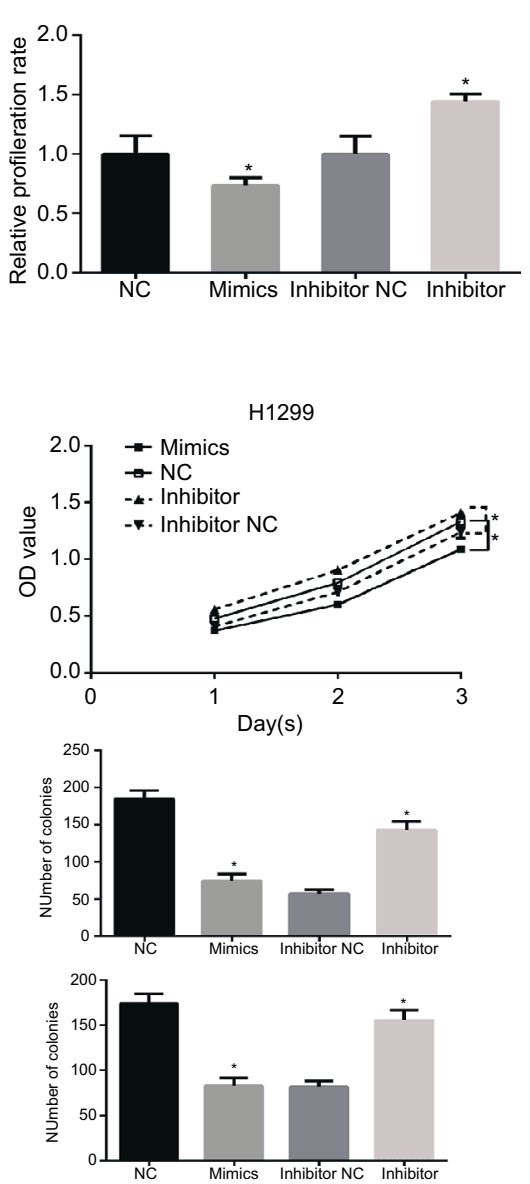

Figure I miR-4458 inhibited cell proliferation in NSCLC cells.

Notes: (A) Relative expression level of miR-4458 in four NSCLC cell lines (A549, HI299, HCC827, and PC9) and one normal lung cell (HBE). (B) The expression of miR4458 after 48 hours of transfection with NC, mimics, inhibitor NC, and inhibitor by qRT-PCR. (C) The rate of cell growth was measured by Edu assay. (D) CCK-8 assay confirmed that miR-4458 inhibited cell proliferation in NSCLC cells. (E) The ability of colony formation was reduced with the overexpression of miR-4458 and enhanced with knockdown of miR-4458. $* P<0.05$, **P<0.01, *** $P<0.001$.

Abbreviations: CCK-8, cell-counting kit-8; Edu, 5-ethynyl-2'-deoxyuridine; miR-4458, microRNA-4458; NC, negative control; NSCLC, non-small-cell lung cancer. 
tion of NSCLC cells compared with the NC group (Figure 2B). To determine the effect of miR-4458 on EMT, we performed Western blotting to evaluate the expression of EMT biomarkers (E-cadherin, N-cadherin, and Snail) after 48 hours of transfection. The findings revealed that miR4458 significantly upregulated the expression of E-cadherin and downregulated the level of $\mathrm{N}$-cadherin and Snail. With knockdown of miR-4458, E-cadherin was reduced while $\mathrm{N}$-cadherin and Snail were increased (Figure 2C). These results suggested that miR-4458 played a pivotal role in the process of migration and EMT.

\section{miR-4458 inhibited migration and the EMT process via PI3K/AKT pathway in NSCLC cells}

$\mathrm{PI} 3 \mathrm{~K} / \mathrm{AKT}$ pathway plays a key role in promoting migration and the EMT process in NSCLC cells. To investigate whether miR-4458 could reduce migration and the EMT process through PI3K/AKT signaling pathway, Western blot assay was carried out. The results indicated that overexpression of miR-4458 obviously reduced the level of p-AKT in NSCLC cells. Consistently, knockdown of miR-4458 led to a robust increase in the expression of $\mathrm{p}$-AKT compared with control group (Figure 3A). In addition, we cultured NSCLC cells with a PI3K inhibitor (LY294002). The results of transwell assay demonstrated that cells treated with LY294002 inhibited migration compared with control group (Figure 3B). Moreover, our results showed that LY294002 group enhanced the expression of E-cadherin and reduced the level of N-cadherin, Snail, and p-AKT (Figure 3C). These findings showed that miR-4458 was involved in the migration and the EMT process through PI3K/AKT pathway in NSCLC.

\section{Bioinformatics analysis of the targets of miR-4458}

Three publicly available databases (TargetScan, TarBase, and miRwalk) were explored to define the targets of miR-4458 (Figure 4A). One hundred seventy-six common target genes were used for the analysis of GO and pathway enrichment (Figure 4B). After the exploration of LUAD expression profile data from the TCGA database, eight more possible targets (HMGA1, VANGL2, E2F2, STRBP, RRP1B, FAXC, SYT7, and CASK) were screened out (Figure 4C). Notably, we selected HMGA1 to do further research. First, the expression of HMGA1 was upregulated in LUAD group compared with normal group in GSE32863 and GSE10072 datasets (Figure 4D). Second, higher HMGA1 expression was correlated with poor progression of NSCLC from Kaplan-
Meier plotter database (Figure 4E). Finally, CCLE database indicated that HMGA1 was highly expressed in lung cancer cell lines (Figure 4F). The qRT-PCR assay confirmed that HMGA1 was upregulated in four NSCLC cell lines (A549, H1299, HCC827, and PC9) compared with HBE (Figure 4G).

\section{HMGAI enhanced migration and the EMT process in NSCLC cells}

In order to confirm the function of HMGA1 in the migration and EMT process, transwell assay, wound healing assay, and Western blot assay were carried out in NSCLC cells. As shown in Figure 5A, cells transfected with si-HMGA1 showed a significant reduction in migration compared with $\mathrm{NC}$ group. The results of wound healing assay indicated that HMGA1 knockdown inhibited migration in NSCLC cell lines (Figure 5B). The expression of EMT biomarker E-cadherin was increased and EMT biomarker N-cadherin and Snail were reduced after HMGA1 was knocked down (Figure 5C). These findings demonstrated that HMGA1 played an important role in regulating metastasis and EMT process in NSCLC cells.

\section{HMGAI was a direct target of miR-4458}

The predicted binding site of miR-4458 in the 3'-UTR of HMGA1 was obtained from miRNA website (Figure 6A). The results revealed that luciferase activity cotransfected with combinations of miR-4458 and h-HMGA1-MUT was increased compared with combinations of miR-4458 and h-HMGA1-WT (Figure 6B). To confirm the expression of HMGA1 in NSCLC cells, qRT-PCR and Western blot were conducted after 48 hours of transfection with miR-4458 NC, mimics, inhibitor NC, and inhibitor. Overexpression of miR4458 obviously inhibited both mRNA and protein expression of HMGA1. Contrastively, miR-4458 knockdown led to a sharp increase of HMGA1 (Figure 6C, D).

\section{Knockdown of HMGAI abolished the role of downregulated miR-4458 in the process of migration and EMT}

The rescue experiment was performed to demonstrate whether HMGA1 could serve as a controller of miR-4458 in the process of migration and EMT. Here are three groups, which were cells transfected with miR-4458 inhibitor, cells cotransfected with miR-4458 inhibitor and si-HMGA1 NC, and cells cotransfected with miR-4458 inhibitor and si-HMGA1. Transwell assay was conducted after the transfection. Compared with miR-4458 inhibitor transfected cells, the migratory capability of miR-4458 significantly decreased in cells cotransfected with miR-4458 inhibitor and si-HMGA1 (Figure 7A). The results of Western 
A

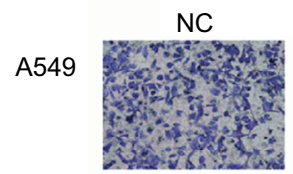

H1299

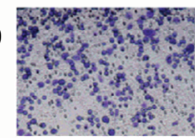

B
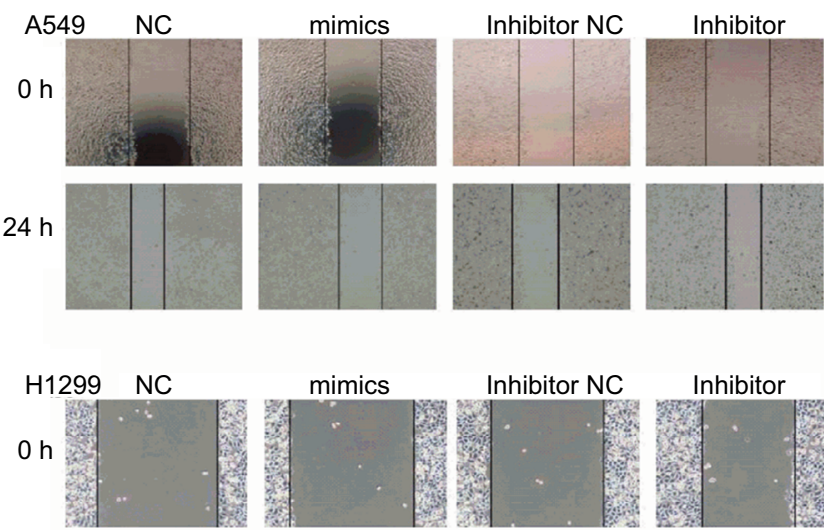

mimics

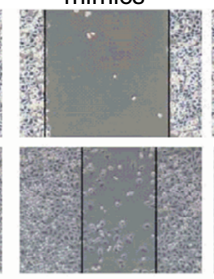

Inhibitor NC
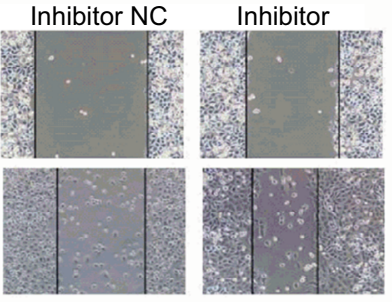

C

A549

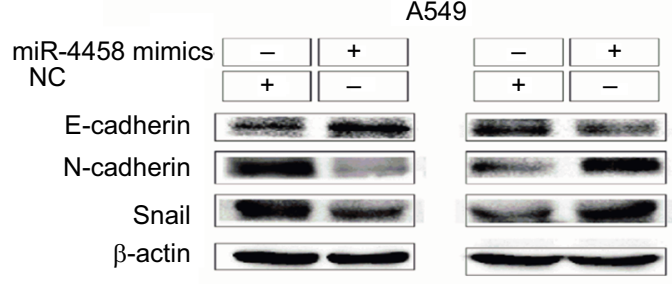

H1299
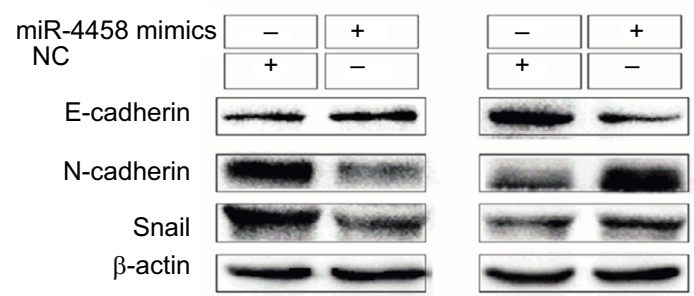

miR-4458 inhibitor Inhibitor NC

miR-4458 inhibitor Inhibitor NC
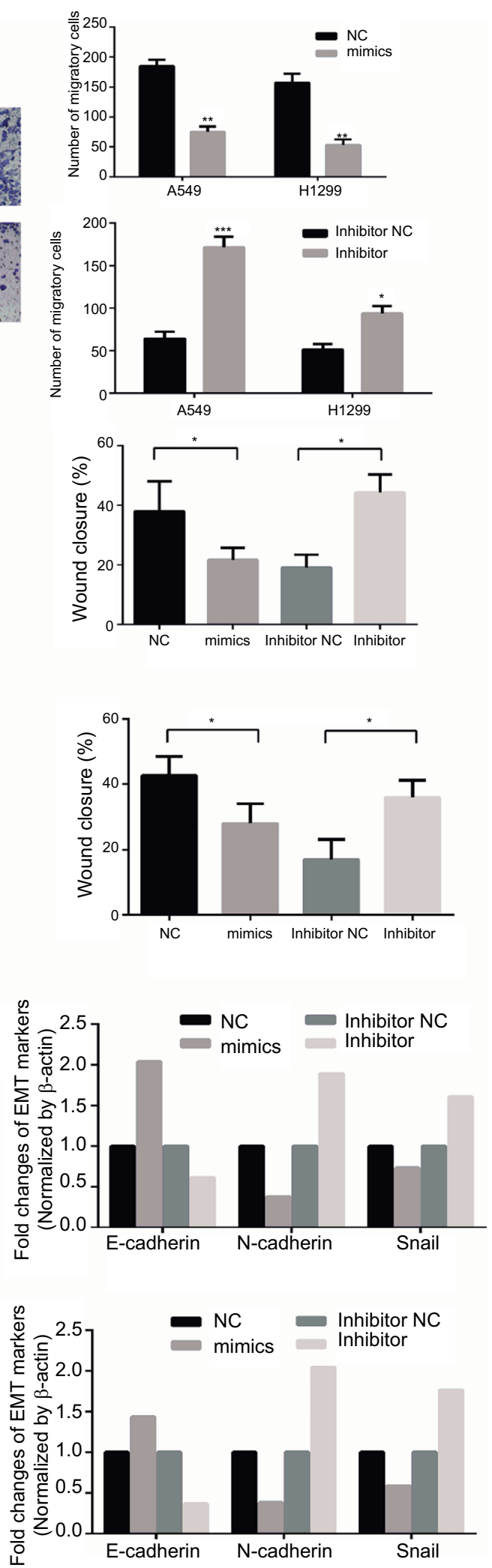

Figure 2 miR-4458 inhibited migration and the EMT process in NSCLC cells

Notes: (A) The results of transwell assay showed that miR-4458 reduced migration ability in NSCLC cells. (B) The results of wound-healing assay showed that miR-4458 inhibited migration ability. (C) Western blot analysis indicated that overexpression of miR-4458 upregulated EMT biomarker E-cadherin and reduced EMT biomarkers $\mathrm{N}$-cadherin and Snail in NSCLC cells. Relatively, E-cadherin was downregulated and $\mathrm{N}$-cadherin and Snail were enhanced with miR-4458 knockdown. $* \mathrm{P}<0.05$, $* * \mathrm{P}<0.0 \mathrm{I}$, $* * * \mathrm{P}<0.001$.

Abbreviations: EMT, epithelial-mesenchymal transition; miR-4458, microRNA-4458; NC, negative control; NSCLC, non-small-cell lung cancer. 
A

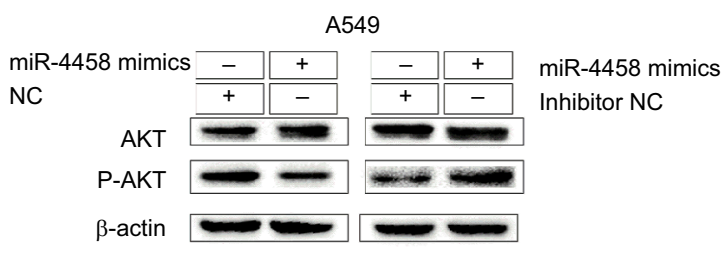

H1299

miR-4458 mimics

NC
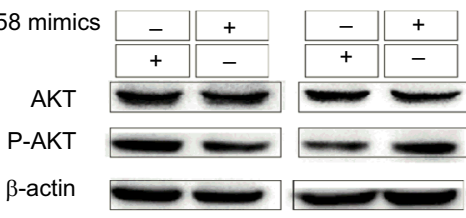

B

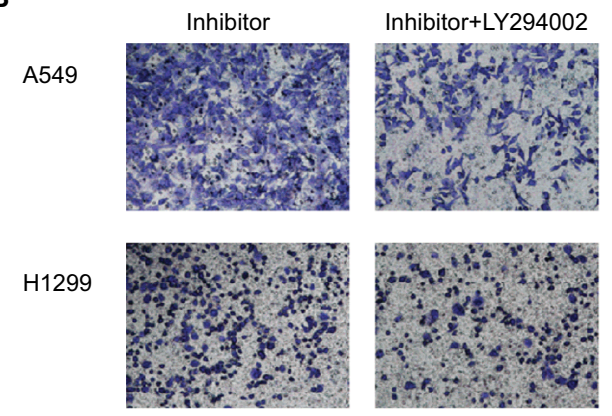

C

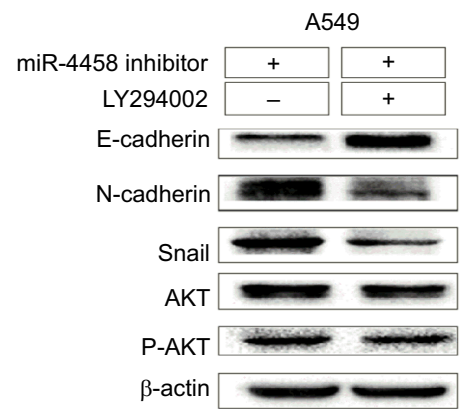

H1299

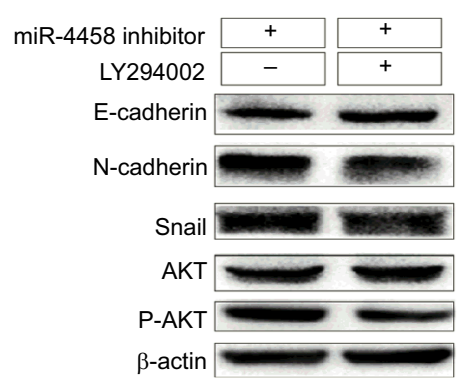

miR-4458 mimics Inhibitor NC

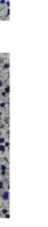

.
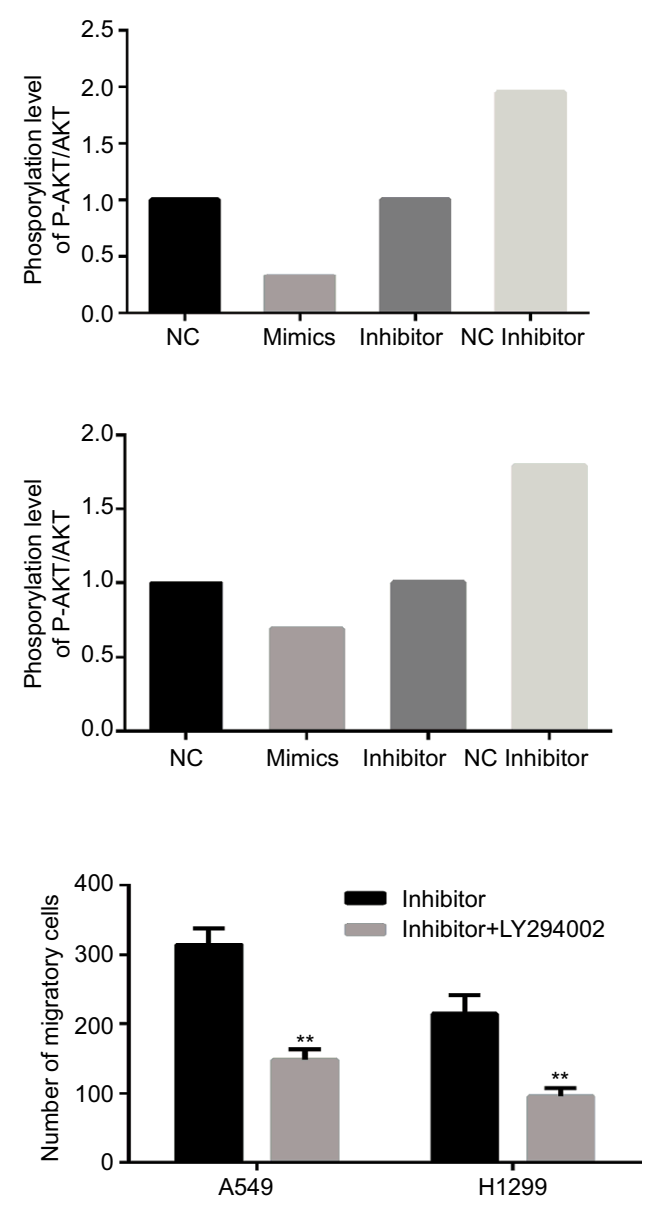

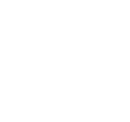


A

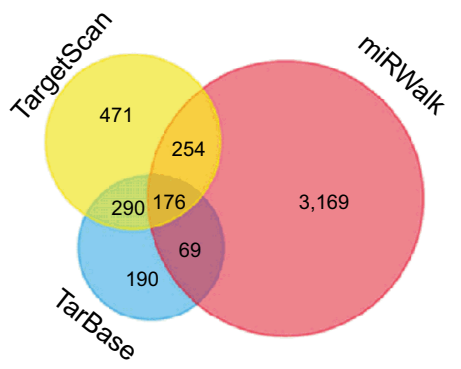

C

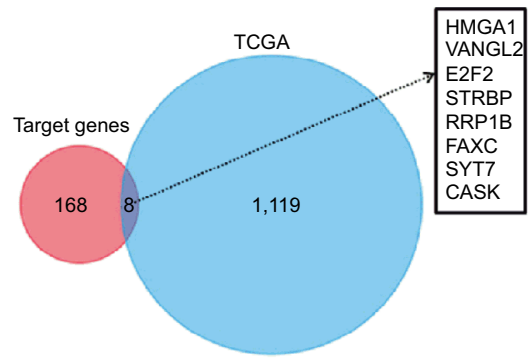

D
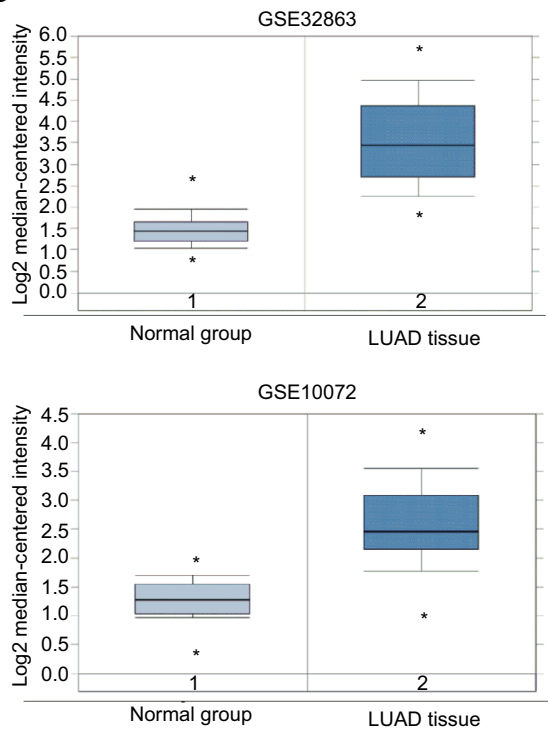

F

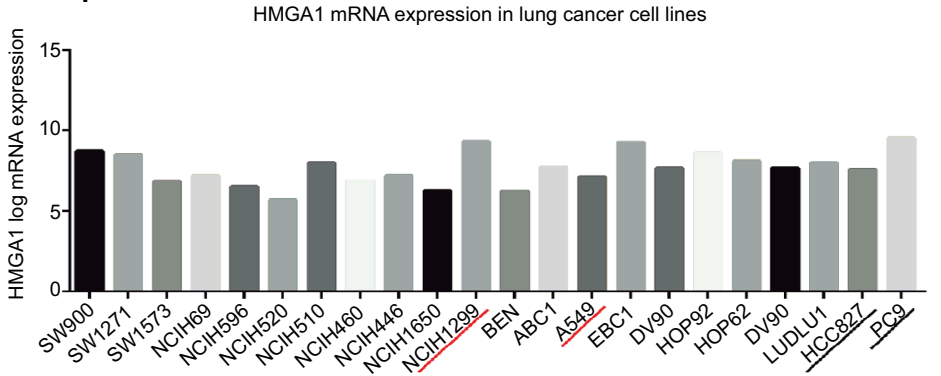

B
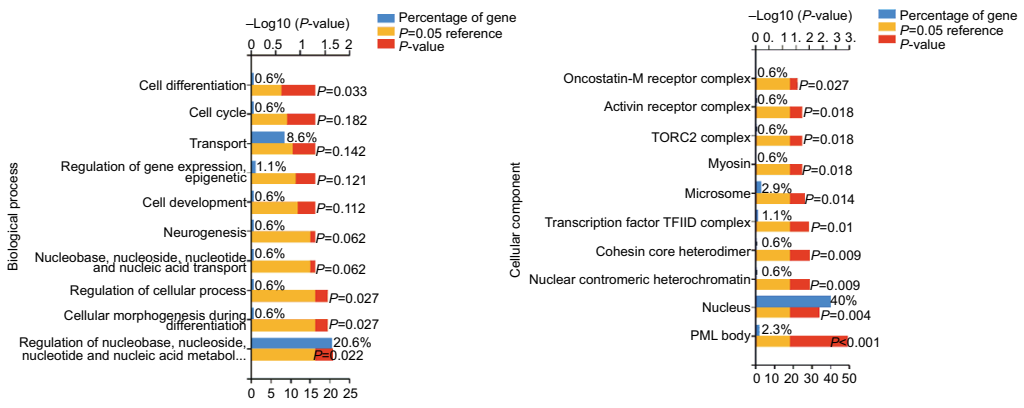

Percentage of gene
$P=0.05$ reference
$P$-value

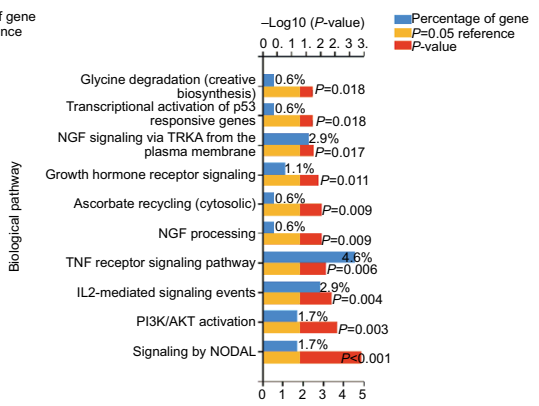

E
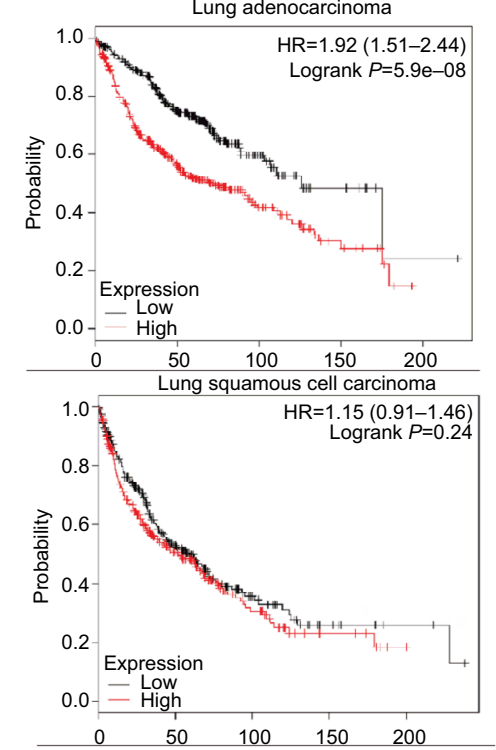

G

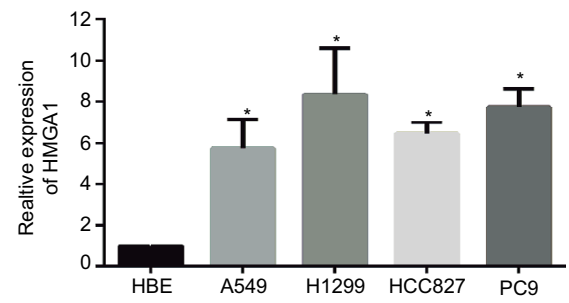

Figure 4 Bioinformatics analysis of the targets of miR-4458.

Notes: (A) Venn chart of common target genes from three publicly available databases including TargetScan, TarBase, and miRwalk. (B) GO analysis included biological processes, cellular component, molecular function, and pathway enrichment analysis for 176 common targets. (C) Venn chart of 8 more possible targets from I76 common target genes and TCGA database. (D) The expression of HMGAI between LUAD and normal group in GSE32863 and GSEI0072 datasets. (E) Analysis of the OS about HMGAI from Kaplan-Meier plotter database. (F) The expression of HMGAI in lung cancer cell lines from CCLE database. (G) The expression of HMGAI between four NSCLC cell lines (A549, HI299, HCC827, and PC9) and one normal lung cell (HBE) by qRT-PCR. $* P<0.05$, $* * P<0.01$.

Abbreviations: GO, Gene Ontology; miR-4458, microRNA-4458; NC, negative control; OS, overall survival. 
A
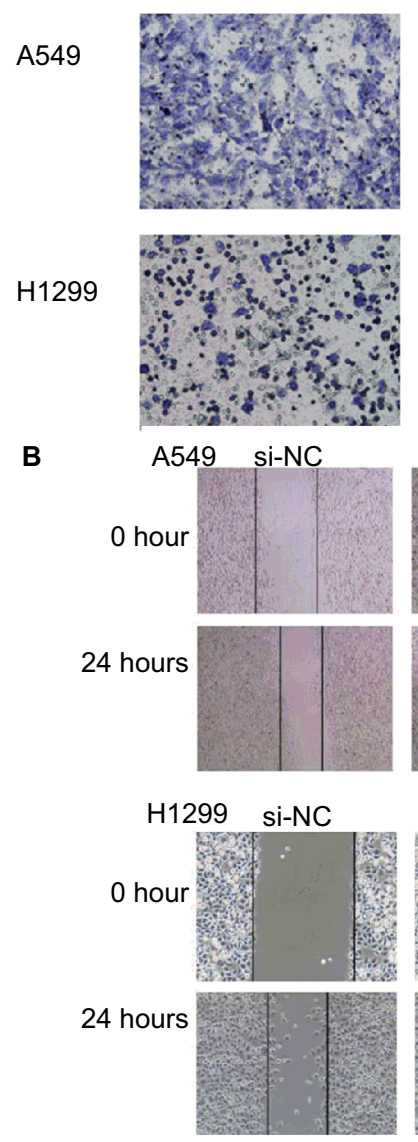

C

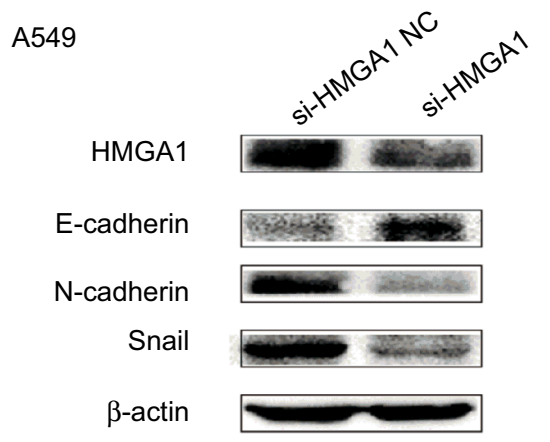

$\mathrm{H} 1299$

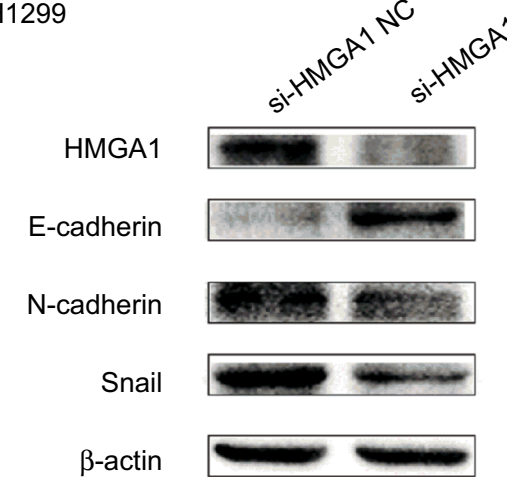

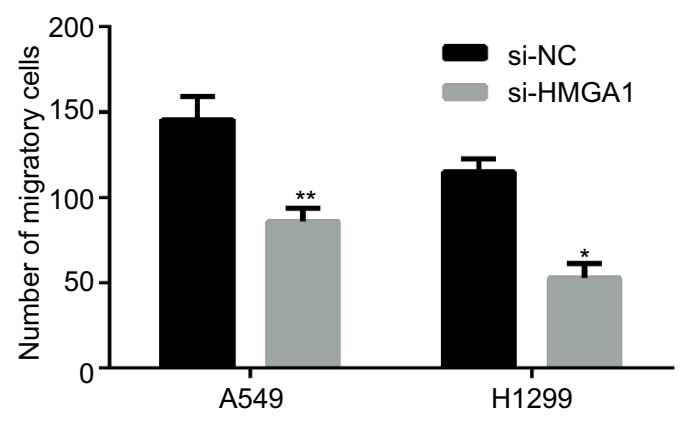
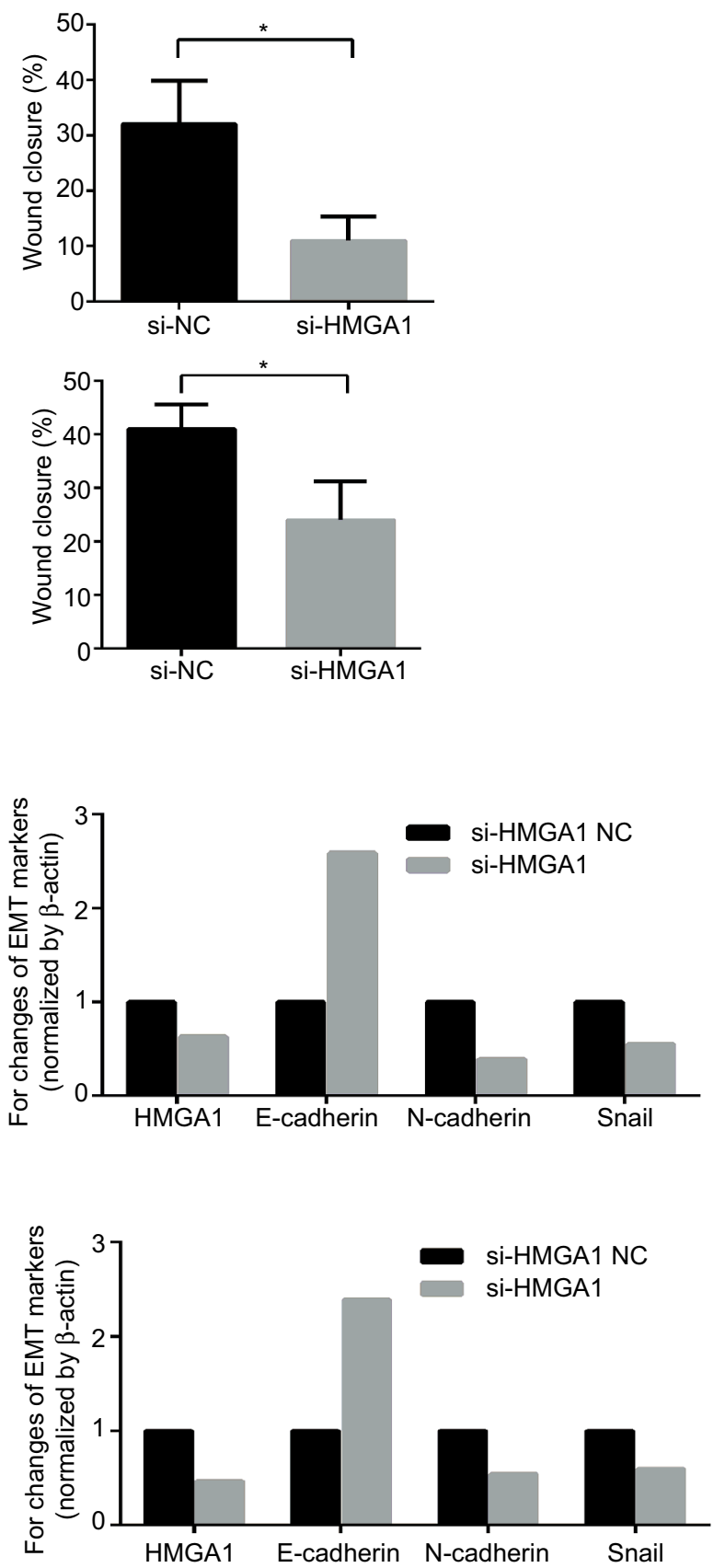

Figure 5 HMGAI enhanced migration and the EMT process in NSCLC cells.

Notes: (A) The results of transwell assay showed that downregulation of HMGAI reduced migration capacity in NSCLC cells. (B) The results of wound-healing assay showed that downregulation of HMGAI inhibited migration capacity in NSCLC cells. (C) Western blot assay indicated that EMT biomarker E-cadherin was upregulated and $\mathrm{N}$-cadherin and Snail were downregulated after knockdown of HMGAI. *P<0.05, **P $<0.01$.

Abbreviations: EMT, epithelial-mesenchymal transition; miR-4458, microRNA-4458; NC, negative control; NSCLC, non-small-cell lung cancer. 
A

HMGA1 3' UTR 5' ...UCCUCUGUUCACAAACUACCUCU... III IIIIIII has-miR-4458 $3^{\prime} \quad$ AAGAAGGUGUGGAUGGAGA
B

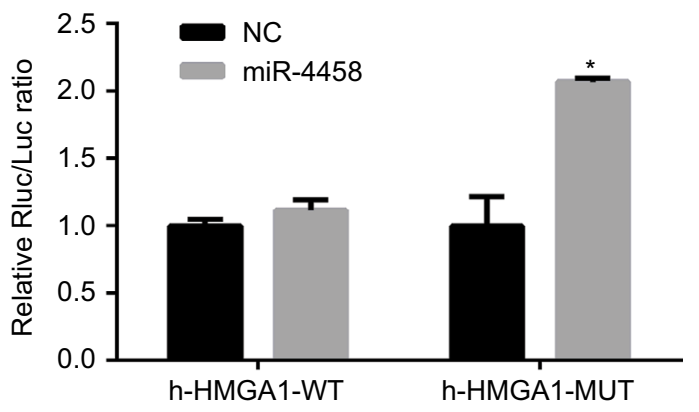

C
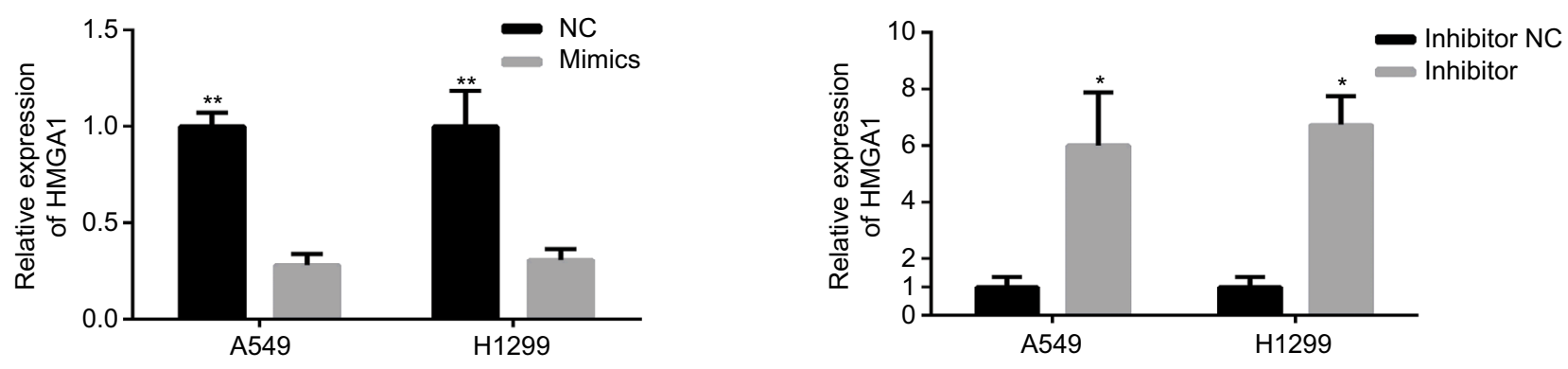

D

A549

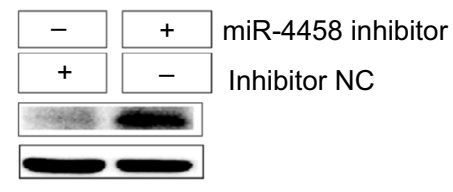

H1299
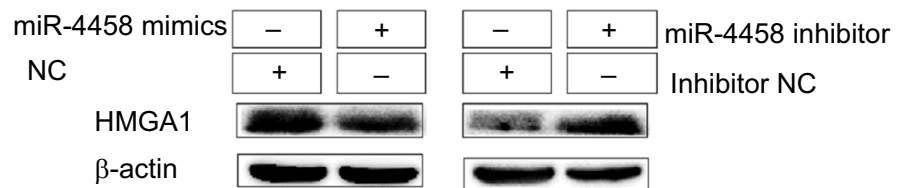

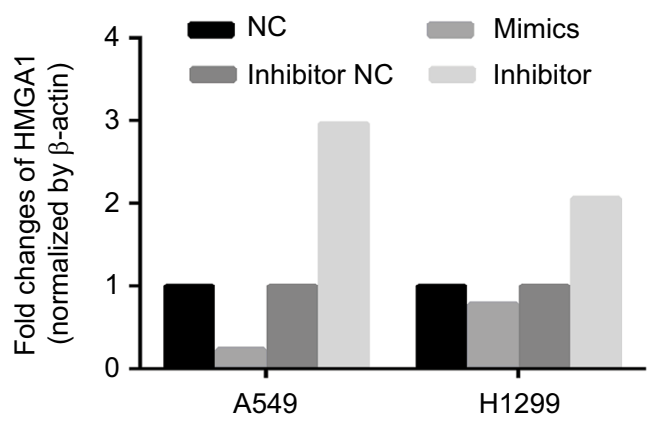

Figure 6 HMGAI was a direct target of miR-4458.

Notes: (A) The predicted binding site of miR-4458 in the 3'-UTR of HMGAI from miRNA website. (B) The analysis of luciferase activity after different combinations of miR4458, miR-NC, h-HMGAI-WT, and h-HMGAI-MUT. (C) The expression of HMGAI in NSCLC cells after 48 hours of transfection with miR-4458 NC, mimics, inhibitor NC, and inhibitor. (D) Western blot analysis of HMGAI in NSCLC cells after 48 hours of transfection with miR-4458 NC, mimics, inhibitor NC, and inhibitor. $* \mathrm{P}<0.05$, $* * \mathrm{P}<0.0 \mathrm{I}$. Abbreviations: miR-4458, microRNA-4458; NC, negative control; NSCLC, non-small-cell lung cancer.

blot assay showed that the expression of E-cadherin increased in cells cotransfected with miR-4458 inhibitor and si-HMGA1. Moreover, the expression of N-cadherin and Snail reduced under the same comparison (Figure 7B). Taken together, these findings showed that miR-4458 inhibited migration and EMT process depending on the regulation of HMGA1 in NSCLC.

\section{Discussion}

It is well known that miRNAs play an important role in the diagnosis and prognosis via binding to $3^{\prime}$-UTR of targets in various malignant neoplasm. ${ }^{5,6}$ In spite of the increasing amount of miRNAs in the development of tumorigenesis, the specific functional molecular mechanism remains unclear. miR-4458 is first detected to be a tumor suppressor in hepatocellular carcinoma. ${ }^{8}$ Moreover, overexpression of miR-4458 suppresses glycolysis and lactate production via targeting hexokinase 2 in colon cancer. ${ }^{9}$ Emerging evidence indicates that miR-4458 inhibits proliferation by directly targeting CCND1 and Lin28B in lung cancer, ${ }^{10,11}$ which is in accordance with the results of cell growth in our study. In the present research, we confirmed that miR-4458 inhibited cell proliferation (Figure 1C, D) and colony formation ability in vitro (Figure 1E). In addition, the expression of the miR4458 was downregulated in NSCLC cell lines by qRT-PCR (Figure 1A). Thus, the results suggest that miR-4458 might inhibit proliferation and colony formation in NSCLC cells. 
A

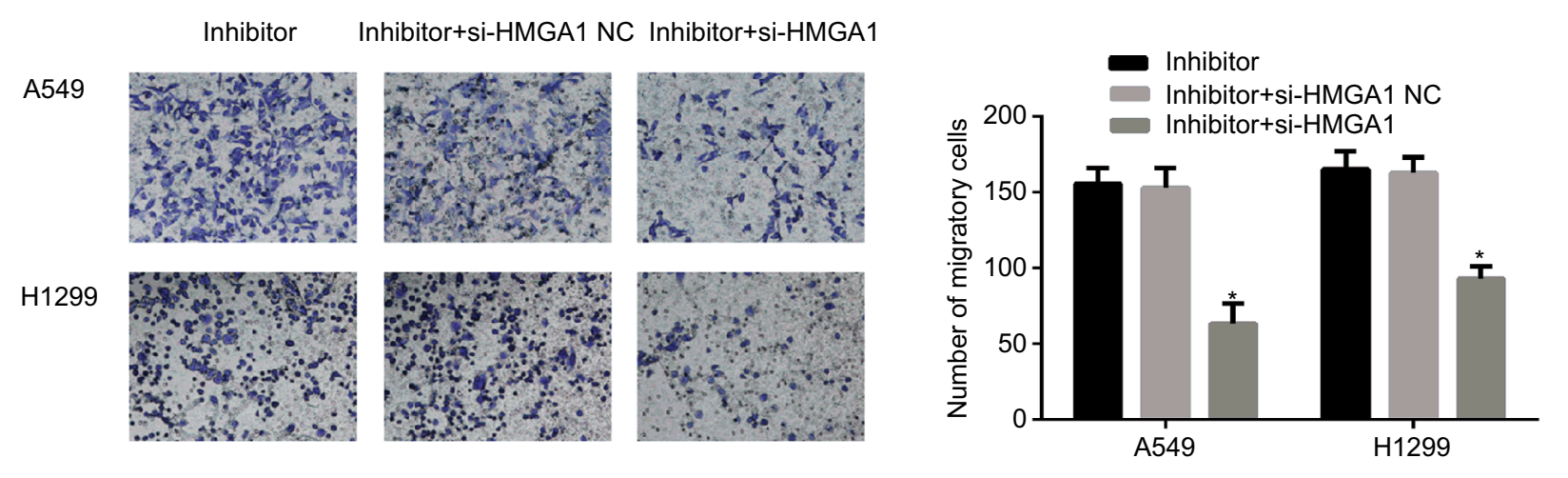

B

A549
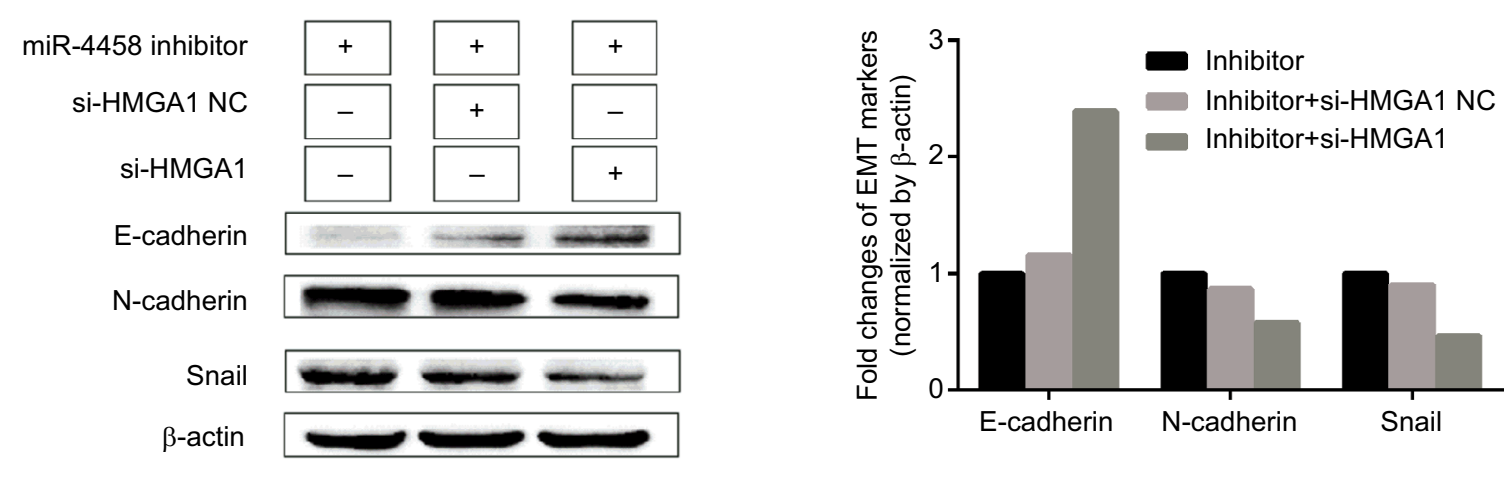

H1299
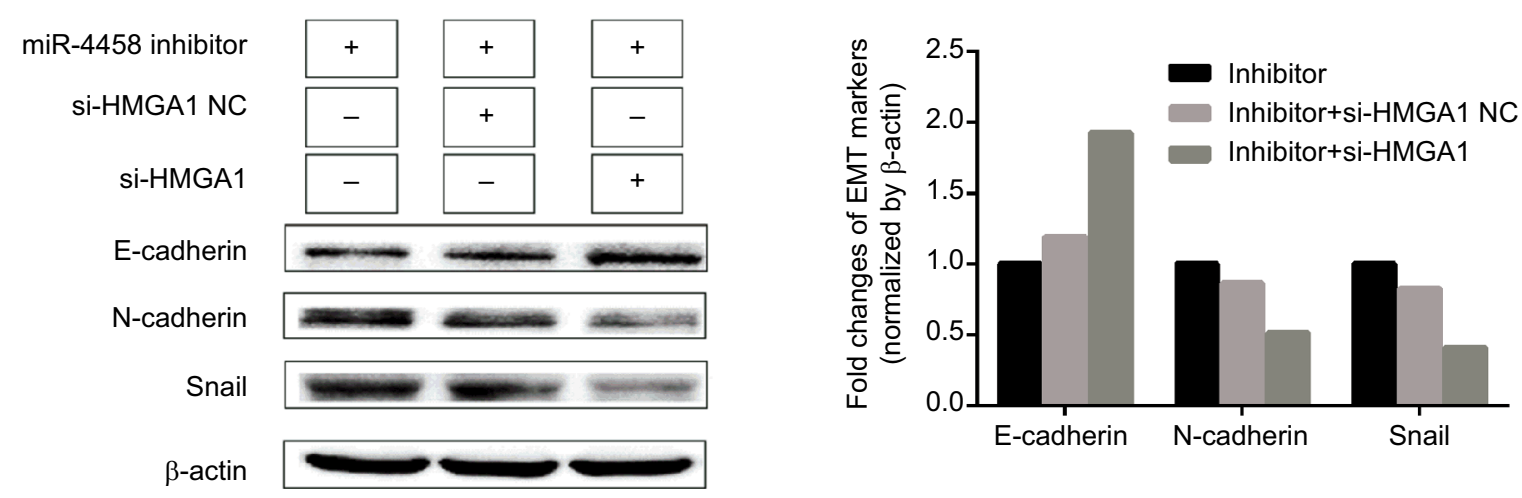

Figure 7 Knockdown of HMGAI abolished the role of downregulated miR-4458 in the process of migration and EMT.

Notes: (A) A549 and HI299 were cotransfected with miR-4458 inhibitor, si-HMGAI NC and si-HMGAI, and the transwell results were shown after 48 hours of transfection. (B) Western blot analysis was carried out after 48 hours of transfection.

Abbreviations: EMT, epithelial-mesenchymal transition; miR-4458, microRNA-4458; NC, negative control.

EMT serves as an important event in the tumor metastasis progression. ${ }^{21-23}$ In the present study, for the first time, we found that miR-4458 suppressed migration and EMT biomarkers in NSCLC cells. First, we performed transwell assay and wound-healing assay to make sure the role of miR-4458 in the development of migration in NSCLC cell. The results showed that miR-4458 knockdown enhanced the number of migratory cells and the width of scratch compared with NC group (Figure 2A, B). Notably, we further conducted the experiment in the analysis of EMT process. Downregulation 
of E-cadherin and overexpression of N-cadherin and Snail are considered as key elements in EMT process. ${ }^{24,25}$ The results discovered that miR-4458 influenced the expression of EMT biomarkers by Western blotting. Overexpression of miR-4458 significantly increased the expression of E-cadherin and reduced the level of $\mathrm{N}$-cadherin and Snail. In contrast, E-cadherin was reduced while N-cadherin and Snail were increased with miR-4458 knockdown (Figure 2C). The results clearly indicate that miR-4458 negatively mediates cell migration and process of EMT through regulating E-cadherin, N-cadherin, and Snail. PI3K/AKT is a pivotal signal pathway in malignant tumor progression and is associated with cell proliferation, migration, and EMT process. ${ }^{26-29}$ In the present study, further studies were carried out to confirm the role of PI3K/AKT pathway in the molecular mechanism of miR-4458. First, the results of Western blot indicated that the level of p-AKT was reduced in NSCLC cells with overexpression of miR-4458. Meanwhile, miR-4458 knockdown enhanced the expression of p-AKT compared with control group (Figure 3A). Second, NSCLC cells were cultured with a PI3K inhibitor (LY294002) in order to explore the further mechanism. The results of transwell assay with miR-4458 knockdown were abolished by LY294002 (Figure 3B). Moreover, our results showed that LY294002 group enhanced the expression of E-cadherin and reduced the level of N-cadherin, Snail, and p-AKT (Figure 3C). Notably, the results of bioinformatics analysis with 176 common targets suggested that PI3K/AKT activation might be involved in the biological pathway of miR-4458 (Figure 4B). These findings show that miR-4458 might play an important role in the migration and EMT process through the PI3K/ AKT pathway in NSCLC.

Recently, a large number of bioinformatics analysis methods have been used to explore the molecular mechanism of laryngeal cancer with the advantage of promoting credibility and reliability. ${ }^{30-32}$ For the analysis of bioinformatics methods in our study, we screened out 176 common target genes from three databases (TargetScan, TarBase, and miRwalk, Figure 4A). Then, we carried out GO (biological processes, cellular component, molecular function) and pathway enrichment analysis by Funrich software (Figure 4B). Additionally, the LUAD database from TCGA was explored to confirm the more possible targets. Finally, eight targets (HMGA1, VANGL2, E2F2, STRBP, RRP1B, FAXC, SYT7, and CASK) were discovered (Figure 4C). Notably, we selected HMGA1 to do further bioinformatics analysis. The results of Oncomine database suggested that the expression of HMGA1 in LUAD tissues was significantly upregulated compared with normal group from two Gene Expression Omnibus datasets
(Figure 4D). Higher HMGA1 expression was correlated with poor progression of both lung adenocarcinoma and lung squamous cell carcinoma from Kaplan-Meier plotter database (Figure 4E). The level of HMGA1 in lung cancer cells was obtained from CCLE database (Figure 4F). These bioinformatics analysis results indicate that HMGA1 may be involved in the tumorigenesis and progression of NSCLC as a potential target of miR-4458. HMGA1 functions as a regulator of the chromatin structure via direct binding to A/Trich DNA sequences. ${ }^{12}$ Accumulating evidence indicates that HMGA1 plays a carcinogenic role in various cancer process, including cell proliferation, cell cycle, and metastasis. ${ }^{13-16}$ In NSCLC, HMGA1 is correlated with the malignant status and prognosis. ${ }^{17}$ Additionally, HMGA1 leads to the promotion of EMT in basal-like breast cancer. ${ }^{18}$ In our study, we explored the function of HMGA1 in the process of migration and EMT. For transwell assay, knockdown of HMGA1 reduced migration capacity in NSCLC cells (Figure 5A). Similarly, wound-healing assay showed that knockdown of HMGA1 inhibited migration capacity (Figure 5B). Western blot assay indicated that EMT biomarker E-cadherin was upregulated and N-cadherin and Snail were downregulated after knockdown of HMGA1 (Figure 5C).

It has been demonstrated that multiple miRNAs could target the same gene. In lung adenocarcinoma cells, miR26a had a negative correlation with HMGA1. ${ }^{19}$ In breast cancer, miR-625 suppressed cell growth and migration via targeting HMGA $1 .{ }^{20}$ However, it is the first time to report that HMGA1 is a direct target of miR-4458 in NSCLC. In our study, we revealed the regulatory relationship of miR-4458 and HMGA1 in NSCLC cells. The luciferase assay confirmed that miR-4458 was directly binding to the 3 -UTR of HMGA1 (Figure 6B). In order to confirm the expression of both mRNA and protein in NSCLC cells, we carried out qRT-PCR and Western blot assay to underpinned the results (Figure 6C, D). Mechanistically, the rescue assay revealed that knockdown of HMGA1 abolished the role of downregulated miR-4458 in the migration and EMT process (Figure 7).

\section{Conclusion}

In summary, our study demonstrated that miR-4458 inhibited proliferation and migration in NSCLC cells. In addition, miR4458 significantly suppressed the progression of metastasis and EMT via the PI3K/AKT pathway. Moreover, miR-4458 participated in the process of migration and EMT process via directly binding to the $3^{\prime}$-UTR of HMGA1. Thus, our results suggest that miR-4458 might be a potential therapeutic target for NSCLC treatment. 


\section{Acknowledgment}

This work was supported by the National Natural Science Foundation of China (Grant No. 81771867, No. 81771868).

\section{Disclosure}

The authors report no conflicts of interest in this work.

\section{References}

1. Torre LA, Bray F, Siegel RL, Ferlay J, Lortet-Tieulent J, Jemal A. Global cancer statistics, 2012. CA Cancer J Clin. 2015;65(2):87-108.

2. Longo-Sorbello GS, Chen B, Budak-Alpdogan T, Bertino JR. Role of pemetrexed in non-small cell lung cancer. Cancer Invest. 2007;25(1):59-66.

3. Wood DE, Kazerooni EA, Baum SL, et al. Lung cancer screening, version 3.2018, NCCN clinical practice guidelines in oncology. $J$ Natl Compr Canc Netw. 2018;16(4):412-441.

4. Saintigny P, Burger JA. Recent advances in non-small cell lung cancer biology and clinical management. Discov Med. 2012;13(71):287-297.

5. Bartel DP. MicroRNAs: genomics, biogenesis, mechanism, and function. Cell. 2004;116(2):281-297.

6. Chen CZ. MicroRNAs as oncogenes and tumor suppressors. $N$ Engl $J$ Med. 2005;353(17):1768-1771.

7. Kang W, Huang T, Zhou Y, et al. miR-375 is involved in Hippo pathway by targeting YAP1/TEAD4-CTGF axis in gastric carcinogenesis. Cell Death Dis. 2018;9(2):92.

8. Tang D, Sun B, Yu H, Yang Z, Zhu L. Tumor-suppressing effect of miR-4458 on human hepatocellular carcinoma. Cell Physiol Biochem. 2015;35(5):1797-1807.

9. Qin Y, Cheng C, Lu H, Wang Y. miR-4458 suppresses glycolysis and lactate production by directly targeting hexokinase 2 in colon cancer cells. Biochem Biophys Res Commun. 2016;469(1):37-43.

10. Bao L, Wang L, Wei G, Wang Y, Wuyun G, Bo A. Role of microRNA-4458 in patients with non-small-cell lung cancer. Oncol Lett. 2016;12(5): 3958-3966.

11. Liu CH, Lv DS, Li M, Sun G, Zhang XF, Bai Y. MicroRNA-4458 suppresses the proliferation of human lung cancer cells in vitro by directly targeting Lin28B. Acta Pharmacol Sin. 2017;38(9):1297-1304.

12. Fusco A, Fedele M. Roles of HMGA proteins in cancer. Nat Rev Cancer. 2007;7(12):899-910.

13. Chiappetta G, Tallini G, de Biasio MC, et al. Detection of high mobility group I HMGI(Y) protein in the diagnosis of thyroid tumors: $\mathrm{HMGI}(\mathrm{Y})$ expression represents a potential diagnostic indicator of carcinoma. Cancer Res. 1998;58(18):4193-4198.

14. Huang R, Huang D, Dai W, Yang F. Overexpression of HMGA1 correlates with the malignant status and prognosis of breast cancer. Mol Cell Biochem. 2015;404(1-2):251-257.

15. Lin SY, Peng F. Association of SIRT1 and HMGA1 expression in nonsmall cell lung cancer. Oncol Lett. 2016;11(1):782-788.
16. Zhong J, Liu C, Zhang QH, et al. TGF- $\beta 1$ induces HMGA1 expression: the role of HMGA1 in thyroid cancer proliferation and invasion. Int $J$ Oncol. 2017;50(5):1567-1578.

17. Zhang Z, Wang Q, Chen F, Liu J. Elevated expression of HMGA1 correlates with the malignant status and prognosis of non-small cell lung cancer. Tumour Biol. 2015;36(2):1213-1219.

18. Pegoraro S, Ros G, Piazza S, et al. HMGA1 promotes metastatic processes in basal-like breast cancer regulating EMT and stemness. Oncotarget. 2013;4(8):1293-1308.

19. Sekimoto N, Suzuki A, Suzuki Y, Sugano S. Expression of miR-26a exhibits a negative correlation with HMGA1 and regulates cancer progression by targeting HMGA1 in lung adenocarcinoma cells. Mol Med Rep. 2017;15(2):534-542.

20. Zhou WB, Zhong CN, Luo XP, et al. miR-625 suppresses cell proliferation and migration by targeting HMGA1 in breast cancer. Biochem Biophys Res Commun. 2016;470(4):838-844.

21. Larue L, Bellacosa A. Epithelial-mesenchymal transition in development and cancer: role of phosphatidylinositol 3' kinase/AKT pathways. Oncogene. 2005;24(50):7443-7454.

22. Yilmaz M, Christofori G. EMT, the cytoskeleton, and cancer cell invasion. Cancer Metastasis Rev. 2009;28(1-2):15-33.

23. Qureshi R, Arora H, Rizvi MA. EMT in cervical cancer: its role in tumour progression and response to therapy. Cancer Lett. 2015;356(2): 321-331.

24. Henderson V, Smith B, Burton LJ, Randle D, Morris M, Odero-Marah VA. Snail promotes cell migration through PI3K/AKT-dependent Rac1 activation as well as PI3K/AKT-independent pathways during prostate cancer progression. Cell Adh Migr. 2015;9(4):255-264.

25. Huber MA, Kraut N, Beug H. Molecular requirements for epithelialmesenchymal transition during tumor progression. Curr Opin Cell Biol. 2005;17(5):548-558.

26. Fresno Vara JA, Casado E, de Castro J, Cejas P, Belda-Iniesta C, González-Barón M. PI3K/Akt signalling pathway and cancer. Cancer Treat Rev. 2004;30(2):193-204.

27. Fu QF, Liu Y, Fan Y, et al. Alpha-enolase promotes cell glycolysis, growth, migration, and invasion in non-small cell lung cancer through FAK-mediated PI3K/AKT pathway. J Hematol Oncol. 2015;8:22.

28. Danielsen SA, Eide PW, Nesbakken A, Guren T, Leithe E, Lothe RA Portrait of the PI3K/AKT pathway in colorectal cancer. Biochim Biophys Acta. 2015;1855(1):104-121.

29. Xu W, Yang Z, Lu N. A new role for the PI3K/Akt signaling pathway in the epithelial-mesenchymal transition. Cell Adh Migr. 2015;9(4): 317-324.

30. Zhang HW, Sun XF, He YN, Li JT, Guo XH, Liu H. Bioinformatics analysis of breast cancer bone metastasis related gene-CXCR4. Asian Pac J Trop Med. 2013;6(9):732-738.

31. Vishnubalaji R, Hamam R, Abdulla MH, et al. Genome-wide mRNA and miRNA expression profiling reveal multiple regulatory networks in colorectal cancer. Cell Death Dis. 2015;6(1):e1614.

32. Emmert-Streib F, de Matos Simoes R, Glazko G, et al. Functional and genetic analysis of the colon cancer network. BMC Bioinformatics. 2014;15(Suppl 6):S6.
Cancer Management and Research

\section{Publish your work in this journal}

Cancer Management and Research is an international, peer-reviewed open access journal focusing on cancer research and the optimal use of preventative and integrated treatment interventions to achieve improved outcomes, enhanced survival and quality of life for the cancer patient. The manuscript management system is completely online and includes

\section{Dovepress}

a very quick and fair peer-review system, which is all easy to use. Visit http://www.dovepress.com/testimonials.php to read real quotes from published authors. 